This paper is a postprint of a paper submitted to and accepted for publication in [IET Intelligent Transport Systems] and is subject to Institution of Engineering and Technology Copyright. The copy of record is available at IET Digital Library.

\title{
Impacts of bus stop location and berth number on urban network traffic performance
}

\author{
Mansour Johari, Mehdi Keyvan-Ekbatani, Dong Ngoduy
}

\begin{abstract}
The effects of operational characteristics of the public transport system on the performance of the urban network traffic flow and the public transport system have been widely investigated at the local level. However, to the best of authors' knowledge there is no attempt to investigate these characteristics at the network level. This study bridges this gap through the notion of network macroscopic fundamental diagram (NMFD). In particular, the effects of the bus stop location (i.e. far-side and near-side) and berth number are discussed at the network level through simulating different scenarios in the central business district (CBD) of the city of Christchurch, New Zealand. In consistent with the local level studies, the outputs show that the far-side bus stops result in better network performance (i.e. larger capacity and critical density range) and a lower median for the network average delay of car traffic. The near-side bus stops instead lead to a lower median for the public transport system. The results reveal that increasing the berth number improves the network capacity and median of the network average delay for both modes. Finally, the impacts of the combination of the far-side and near-side bus stop on network performance have been discussed.
\end{abstract}

\section{INTRODUCTION}

Public transport is a key strategy to mitigate the congestion problem in the modern societies. The public transport system requires providing an acceptable service between different points of the network to motivate car users to shift their transportation mode to the public transport system [1]. This yields the importance of the network design for the public transport system. The design studies, mostly consist of optimization procedures that aim to optimize factors like the headway, line spacing, and stop spacing [2-5] through minimizing the agency, users [2-4], and emission [5] costs. Performancerelated studies are also crucial to assess the operation of the existing public transport networks and to provide useful insights to be taken into account in the network design process. In addition, since the operational characteristics of the public transport system impress the interactions between the different transportation modes, the performance studies improve our understanding of the traffic dynamics in the bimodal (i.e. car and bus) urban networks.

Remarkable literature exists discussing how different characteristics of the public transport system like the dwell time, bus stop location, number of berth, etc. affect the public transport performance and also the car traffic. However, this literature mainly includes local level studies and the network level impacts of these characteristics have remained challenging. The network level investigation of theses impacts: (i) makes the assessment of the network performance under different condition and factors possible; (ii) provides useful insights for design studies. The later can be an interesting insight since these factors (i.e. bus stop location, number of berth, etc.) normally have not been applied in design studies.

This paper discusses the network level effects of the bus stop location and number of berth. These factors have been considerably investigated in the local level studies. Typically, there are three types of bus stop locations (i) near-side (located upstream of the intersection), (ii) far-side (located downstream of the intersection), and (iii) mid-block (isolated from the intersections), see Fig. 1. [6] commented that the mid-block bus stop should be avoided since it increases the walking distances and results in a higher number of unauthorized street crossing. Various studies have been conducted

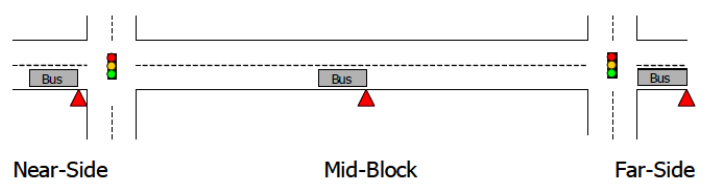

Fig. 1: Bus stops locations [7].

on the impacts of the bus stop location on public transport performance. For instance, [8] showed that on hills and nearside bus stops increase the public transport delay. However, the bus stop location effects on car traffic have not been sufficiently investigated. The initial insights were proposed in [9] through the analytical models under the assumption that the dwell time and distance of bus stop from the intersection do not affect the car traffic. The considered assumption is far from reality and was later contradicted by [10]. [11] showed through a two lane cellular automaton model that the nearside bus stop causes capacity drop if it is installed bellow a critical distance from the intersection. [10] later mentioned a group of factors like the car inflow, green ratio, and length of the signal cycle as the factors which determine the effects of a near-side bus stop on the car traffic. Next, [12] showed that the near-side bus stop can decrease the car delay, while the far-side bus stop can decrease the bus delay. Then, [13] 
analytically showed that the distance between the bus stop and intersection, dwell time, and the capacity of bottleneck created by the bus stop can affect the intersection capacity. Recently, [14] presented that when the inflow is greater than a critical value, the impact of bus stop on local traffic is larger. However, this impact disappeared by increasing the distance of the bus stop from the intersection. [15] also presented that the near-side bus stop is superior when the major concern is bus-carrying capacity. However, as mentioned earlier, the network level investigation on the effects of the near-side and far-side bus stops on the network performance and public transport system is a missing point of the existing literature. Besides, some local level studies resulted in the opposite outputs, while their main concern was the same (e.g. [12] and [15]).

The literature on the effects of the berth number also suffers from the lack of network level investigations. A berth is defined as "the position for a bus to pick up and discharge passengers, including curb bus stops and other types of boarding and discharge facilities" [9]. Number of berth has been in the spotlight of the research community with the fast development of the public transportation system in the recent years. In particular, this is an important problem in the CBD of the populated cities where a bus stop normally serves more than one bus line. However, the literature in this area is still in its infancy. The existing literature discusses the problem of the berth assignment and to a lesser extent the effects of the berth number on the public transport system and traffic condition. For instance, [16] showed through simulation data that the berth number significantly affects the bus speed in the urban roads. Next, [17] presented that the bus delay and the rate at which the bus delay increases with bus inflow decrease as the number of berth increases. [18] later discussed that the rise in the number of berth increases the capacity and speed of the car traffic. Then, [19] showed that the capacity of the link considerably increases as the number of berth increase up to three, but for the berth number higher than three the capacity does not experience a considerable raise. This result is exactly in consistent with the effects of the number of berth on the capacity of link which was presented by [9].

To bridge this gap (i.e. lack of network level investigation on the effects of the operational characteristics of the public transport system), this study discusses bus stop location and berth number impacts on the network performance and public transport system at the network level via the notion of the network macroscopic fundamental diagram (NMFD). To this end, different scenarios are simulated in the CBD of the city of Christchurch, New Zealand. Then, scenarios are compared by studying the 3D-NMFDs and the corresponding contour plot in terms of: the performance in free flow condition, capacity range, and critical density range. The comparison between the network average delay of the different scenarios is also provided to discuss the public transport system performance. Outputs show that the far-side bus stops result in a bigger capacity and critical density range compared to the near-side bus stops. The far-side bus stops also lead to a lower median in the network average delay box plot for the car traffic, while the near-side bus stops lead to a lower median for the public transport system. The results reveal that the raise in the berth number improves the network capacity and median of the network average delay of the car traffic and public transport system. In addition, a comparison between the introduced scenarios for bus stop locations and the current condition of the Christchurch CBD demonstrates that to have the optimal network performance in the real condition, a combination of the far-side and near-side bus stops need to be used.

The remainder of the paper is organized as follows: section 2 presents a brief overview on the macroscopic modelling of urban networks and studies that have conducted on the effects of the bus stop location and berth number using this notion. Section 3 presents the methodology including the NMFD derivation , test-bed description and simulation setup, and the scenarios. This is followed by section 4 which presents the results and discussion. Finally, conclusions are addressed in section 5 .

\section{Macroscopic Modeling of URban Networks}

The NMFD is a reproducible diagram that illustrates the relationships between fundamental traffic variables at the network level see Fig. 2. Macroscopic modelling of urban networks was established and developed by [20-22]. These models were brought again into the spotlight thorough reintroducing and verifying by $[23,24]$.



Fig. 2: The network macroscopic fundamental diagram (NMFD).

Since then, lots of studies have discussed the NMFD existence [25], properties [26], and application [27]. A significant contribution in the NMFD literature is the existence of 3DNMFD which relates the vehicular (passenger) flow in a bimodal network to the car and bus accumulations (densities). The idea of the NMFD for the bi-modal urban networks was introduced in [28]. Then, [29], for the first time, showed through the simulation data that a 3D-NMFD exists for the bi-modal urban networks. The existence of 3D-NMFD was later confirmed through the empirical data [30] see Fig. 3.

Recently, [31] presented the functional form of the 3DNMFD. The suggested function includes two parts. The first part presents an upper bound for travel production. Then, this amount is reduced by a smoothing parameter to mimic 


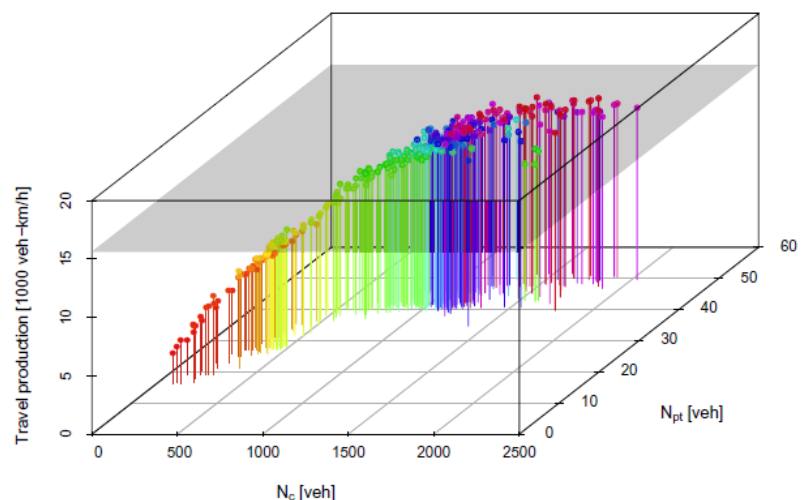

Fig. 3: Empirical 3D-NMFD, the city of Zurich, Switzerland [30].

the interaction between the modes and the heterogeneity of the flow. The 3D-NMFD shape is sensitive to the operational characteristics of the public transport system. This makes it an appropriate tool to discuss the effects of these characteristics at the network level. Firstly, [28] mentioned the bus headway, behavior of offsets, dwell time, bus arrival, bus stop location, number of bus line, type of bus stops, and berth number as the factors which could affect the shape of the NMFD of a bi-modal urban network. Next, [29] presented through the simulation data that a longer dwell time leads to a lower average speed and flow in the NMFD of buses. This attracted the attention of the community that the 3D-NMFD can be applied to study the effects of different factors on the performance of the bi-modal urban networks. [32] presented that the dedicated bus lane and transit signal priority lead to a larger flow capacity in comparison to the scenarios with the mixed traffic and fixed signal timing. Later, [33] showed that the moving bottleneck effect and the capacity reduction, are the major effects of buses on the NMFD of a corridor. Recently, [34] showed via simulation data that the far-side bus stops might results in a bigger critical density range in comparison to the near-side bus stops at the network level.

\section{Methodology}

In this study, first, different scenarios (two, for different bus stop location and three for the berth number) are introduced. Next, the scenarios are simulated in the CBD of Christchurch, New Zealand through the microscopic simulation environment. Then, the 3D-NMFDs of the different scenarios are derived and applied to discuss the effects of the bus stop location and berth number on the network performance. To this end, similar to the applied approach in [32], the obtained 3D-NMFDs are compared in terms of the network capacity and critical density ranges as the city decision makers and practitioners are interested to derive policies and strategies under which the network is performing close to its capacity and critical density ranges [29]. In addition, the network level effects of different scenarios in terms the public transport system and car traffic delay are discussed. Finally, a comparison between the current condition of the CBD with the bus stop location scenarios is provided to discuss the differences between the considered scenarios and the real condition. The following subsections (i) present how the 3D-NMFDs are plotted, (ii) describe the test-bed and simulation setup, and (iii) introduce the considered scenarios.

\section{A. NMFD Derivation}

The NMFD can be derived through the analytical approach (method of cuts), empirical data, and simulation data. The analytical NMFD determines the upper-bond of the corresponding variables, while the empirical and simulated NMFDs show scatters. The loop detector data (flow and density) has been widely employed to derive the empirical or simulated NMFD. Some studies also plotted the NMFD through the total travel time and total travel distance [35] and the trajectory data [36]. In this study, the 3D-NMFD is derived to compare the different scenarios. To do this, detectors collect the data (flow and density) every $90 \mathrm{~s}$ during the simulation. Herein, 3D-NMFD relates the average total flow of the entire network to the average density of cars and average density of buses calculated as follow:

$$
\begin{aligned}
& Q_{\text {total }}(t)=\frac{\sum_{i \in \mathrm{Z}} q_{i}(t) \cdot l_{i}}{\sum_{i \in \mathrm{Z}} l_{i}} \\
& K_{\text {car }}(t)=\frac{\sum_{i \in Z} k_{i}^{\mathrm{c}}(t) \cdot l_{i}}{\sum_{i \in Z} l_{i}} \\
& K_{\text {bus }}(t)=\frac{\sum_{i \in \mathrm{Z}} k_{i}^{\mathrm{b}}(t) \cdot l_{i}}{\sum_{i \in \mathrm{Z}} l_{i}}
\end{aligned}
$$

Where the used notations are presented in Table I. Besides, as mentioned in [29], in order to represent the differences between the different transportation mode's passenger occupancy it is crucial to derive the passenger 3D-NMFD. The passenger 3D-NMFD relates the average total passenger flow in a bi-modal network to the average density of cars and buses within the network. In our study, the passenger flow is calculated using the model presented in [29] as follow:

$$
Q_{\text {total }}^{\mathrm{p}}(t)=h_{\mathrm{c}} Q_{\mathrm{c}}(t)+h_{\mathrm{b}} Q_{\mathrm{b}}(t)
$$

Where the employed notations are shown in Table I.

\section{B. Test-bed Description and Simulation Setup}

The CBD of Christchurch, New Zealand is modeled in microscopic simulation environment Aimsun [37] to explore the scenarios introduced in this study see Fig. 4. To this end, the real origins and destinations (OD), control plans (phases, phase order, etc), and public transport system are employed. The following items indicate the inputs applied to model the network:

- Network geometry

The geometry of the network is modeled based on the aerial map of the CBD and the Google Street View. 
TABLE I: The summary of notations.

\begin{tabular}{ll}
\hline Key & Definition \\
\hline$t$ & Time index \\
$i$ & Link index (links with detector) \\
$Q_{\text {total }}(t)$ & Average total flow at time $t(\mathrm{veh} / \mathrm{hr})$ \\
$Q_{\text {total }}^{\mathrm{p}}(t)$ & Average total passenger flow at time $t(\mathrm{per} / \mathrm{hr})$ \\
$Q_{\mathrm{c}}(t)$ & Average flow of car at time $t(\mathrm{veh} / \mathrm{hr})$ \\
$Q_{\mathrm{b}}(t)$ & Average flow of bus at time $t(\mathrm{veh} / \mathrm{hr})$ \\
$K_{\mathrm{car}}(t)$ & Average density of car at time $t(\mathrm{veh} / \mathrm{km})$ \\
$K_{\mathrm{bus}}(t)$ & Average density of bus at time $t(\mathrm{veh} / \mathrm{km})$ \\
$q_{i}(t)$ & Flow on the link $i$ at time $t(\mathrm{veh} / \mathrm{hr})$ \\
$k_{i}^{\mathrm{c}}(t)$ & The car density on the link $i$ at time $t(\mathrm{veh} / \mathrm{km})$ \\
$k_{i}^{\mathrm{b}}(t)$ & The bus density on the link $i$ at time $t(\mathrm{veh} / \mathrm{km})$ \\
$l_{i}$ & The length of the link $i(\mathrm{~km})$ \\
$h_{\mathrm{c}}$ & Car passenger occupancy $(\mathrm{per} / \mathrm{veh})$ \\
$h_{\mathrm{b}}$ & Bus passenger occupancy $(\mathrm{per} / \mathrm{veh})$ \\
$\mathrm{Z}$ & Set of the measurement links \\
\hline &
\end{tabular}

The origins and destinations (OD) are modeled based on the 2018 CAST model [38] of the CBD. The CAST model is a macroscopic model of the Christchurch that was developed in SATURN [39] by Christchurch city council.

\section{- Traffic signal}

The CBD consists of 98 signalized intersections. A fixed signal timing is considered. Herein, the applied green time for each phase is the average green time of the phase within the considered simulation horizon. The average green time and also the phase order data are gathered from the controller information sheets and the SCATS history viewer provided by the Christchurch city council.

\section{- Public transport system}

The public transport system in the CBD consists of 15 transit lines and 88 bus stops see Fig. 5. All the bus stops are bay bus stops and the mean dwell time is considered $30 \mathrm{~s}$ for each bus stop. The mean dwell time follows a normal distribution with a 33\% standard deviation. Moreover, the real time-table from [40] is considered for all transit lines. It is worth mentioning that the traffic condition is mixed (i.e. no dedicated lane is introduced for the public transport system). Note that, the observed concentration of grey lines in the central part of CBD (Fig. 5) is due to the existence of the bus interchange for indoor bus exchanges within the CBD of the Christchurch.

\section{- Road signs}

The speed limits are also imported in the model. To this end, the speed limit information is gathered from the speed limit map provided by the city council.

- Other model elements

The priority rules and stop signs are also modeled in order to closely mimic the real condition.

Each scenario is simulated by 15 replications with different random seeds to take into account the stochasticity effect of Aimsun. The horizon of the simulation is 2 hours (7:00 AM - 9:00 AM) which is the morning peak period of the Christchurch CBD. The real OD matrices of the CBD are introduced to Aimsun from the 2018 CAST model. During the simulation, the dynamic traffic assignment module is activated to have more real demand distribution in the network [41]. Finally, the loop detectors are installed in the middle of each link (except the short links) to collect the data.

\section{Scenarios}

The public transport system in Christchurch CBD includes 88 bay bus stops. The set of bus stops consists of the 43 mid-block bus stops (located more than $30 \mathrm{~m}$ from the intersections), 34 far-side and 11 near-side bus stops. in case of the bus stop location, two scenarios are defined as follow:

\section{- Far-Side Scenario (FSS)}

This scenario presents the situation that all the bus stops, that are located near to the intersections (less than $30 \mathrm{~m}$ ), are far-side bus stops. To implement this scenario, a near-side bus stop is replaced with a far-side bus stop with the same length and same distance from the intersection. This process is applied to all of the near-side bus stops in the CBD except three bus stops due to the network geometry limitation (e.g. the existence of short link downstream of the intersection).

\section{- Near-Side Scenario (NSS)}

The scenario describes the situation that all the bus stops, that are located near the intersections (less than 30 $\mathrm{m})$, are near-side bus stops. To carry out this scenario, a far-side bus stop is replaced with a near-side bus stop with the same length and same distance from the intersection. Here, 23 of 33 far-side bus stops are turned to the near-side bus stops, while the network geometry does not allow to change the 10 bus stops.

In the public transport system, 48 bus stops serve only one bus line, 17 bus stops serve two bus lines, and 23 bus stops serve three or more bus lines. In this study, the berth number is not assumed more than three since a berth number more than three can not efficiently improve the network performance [42]. Three scenarios are considered to discuss the network level effects of the berth number as follow:

\section{- Berth-1 Scenario (B1S)}

In this scenario, the berth number is assumed to be one in all of the bus stops without taking the number of bus lines that share the bus stop into account. To this end, the length of all bus stops are set as $14 \mathrm{~m}$ [16] in the microscopic simulation environment, while the length of a bus is $12 \mathrm{~m}$. This scenario does not represent a real situation, but is defined to explore the network level effects of the rise in the berth number.

- Berth-2 Scenario (B2S)

This scenario represents the situation that, the number 


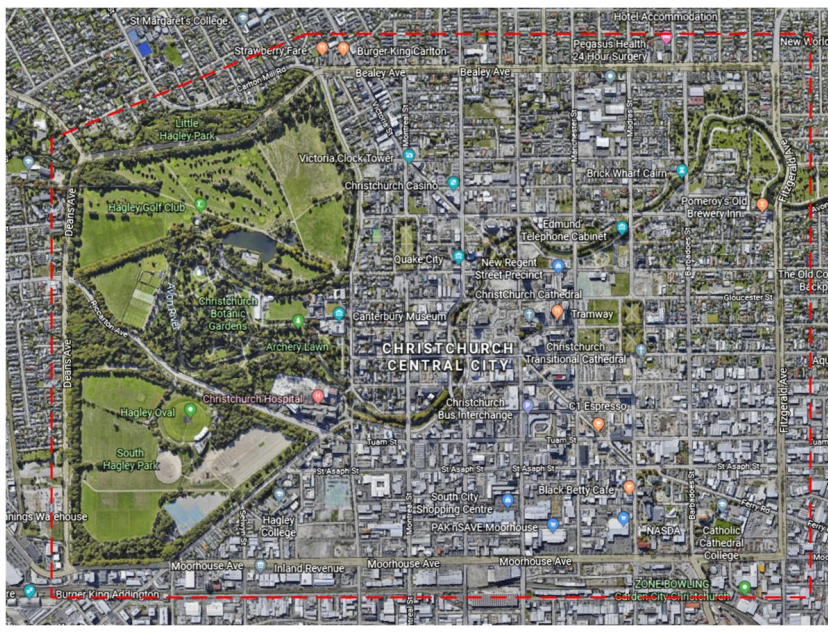

(a)

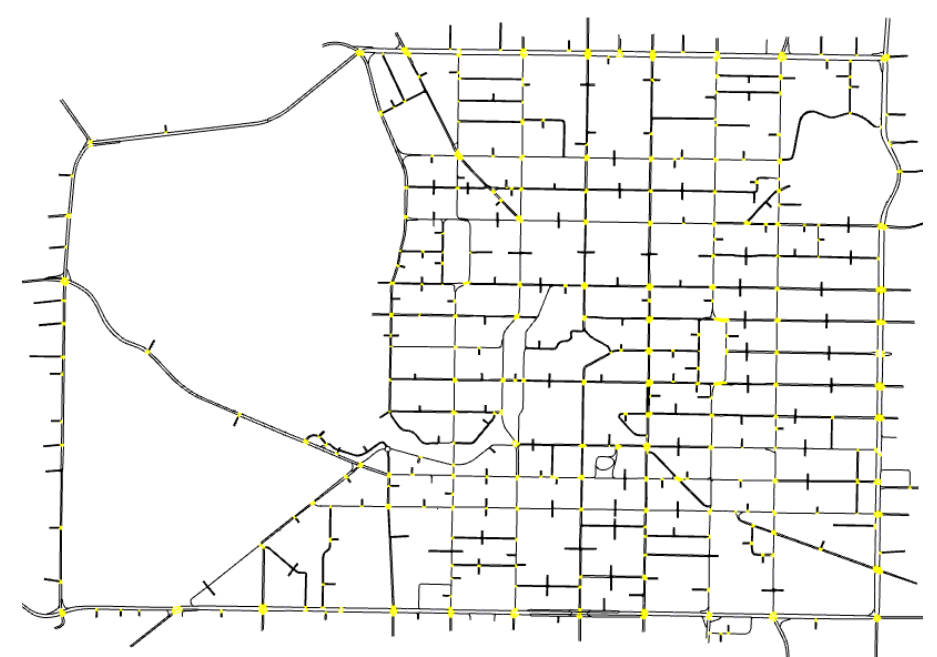

(b)

Fig. 4: (a) Satellite view of Christchurch CBD (b) The CBD network modelled in Aimsun.

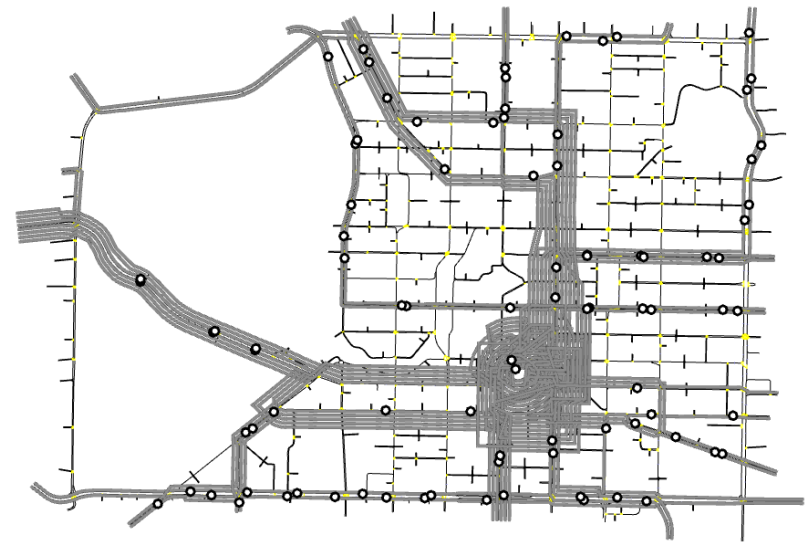

Fig. 5: The gray lines depict the bus lines in the network. The bus stops are shown by the black empty circles.

of berth is one in all the bus stops which serve one bus line, while the berth number is defined two in all the bus stops that serve two or more bus lines. The berth number of two is defined by increasing the length of the bus stop from $14 \mathrm{~m}$ to $28 \mathrm{~m}$.

\section{- Berth-3 Scenario (B3S)}

In this scenario, the berth number is one and two for the bus stops that serve one bus line and two bus lines, respectively. The berth number is assumed three for the bus stops which serve three or more bus lines. To this end, the length of these bus stops is set as $42 \mathrm{~m}$.

\section{RESULTS AND DISCUSSION}

\section{A. 3D-NMFD existence}

This paper applies the notion of the 3D-NMFD to investigate the network level impacts of the bus stop location and berth number on network performance. Therefore, it is crucial to, first, explore the existence of the 3D-NMFD in the corresponding network. Fig.6a presents the 3D-NMFD of the city of Christchurch, New Zealand. As the first remark, Fig.6a confirms the existence of the 3D-NMFD. To provide a more detailed view, Fig.6b presents the corresponding contour plot on the $\left(k_{\text {car }}, k_{\text {bus }}\right)$ plane where the colour bar depicts the different levels of the total flow (veh/hr) in the network. The observed trends and 3D-NMFD shape are in consistent with the simulated and empirical 3D-NMFDs in the existing literature $[29,30]$. The network performs close to its capacity (400 veh/hr) at the car density range of (35$50 \mathrm{veh} / \mathrm{km})$ and bus density range of $(0.5-2 \mathrm{veh} / \mathrm{km})$. The observed ranges shape the optimal operational regime, first introduced by [29], of the Christchurch network and can be applied to design the traffic control strategies or travel demand management policies. Besides, Fig.6b shows that the total flow decreases as car and bus density increases. However, the reduction occurs with different slopes (i.e. higher slope for bus). This means that adding one bus has different effects on network performance in comparison to adding a car [29].

\section{B. Bus stop location}

This subsection presents the discussion on the bus stop location scenarios. In the considered scenarios, it is assumed that almost all the bus stops that are close to the intersections can be either far-side or near-side. However, it is almost impossible to have only one type of bus stop location (i.e. far-side or near side) for bus stops that need to be located near to the intersections in the real urban networks. This happens because of (i) the network geometry limitation like the existence of the short links downstream or upstream of the intersection which has been observed in this study, (ii) considered criteria and the applied guideline in designing the bus stops location. The later can happen in real condition like the situation that a center of the trip generation and attraction exists downstream or upstream of the intersection and the applied guideline forces the designers to place the 


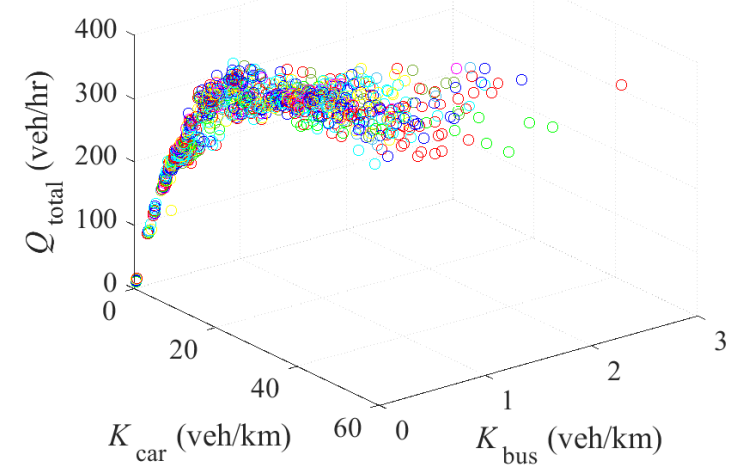

(a)

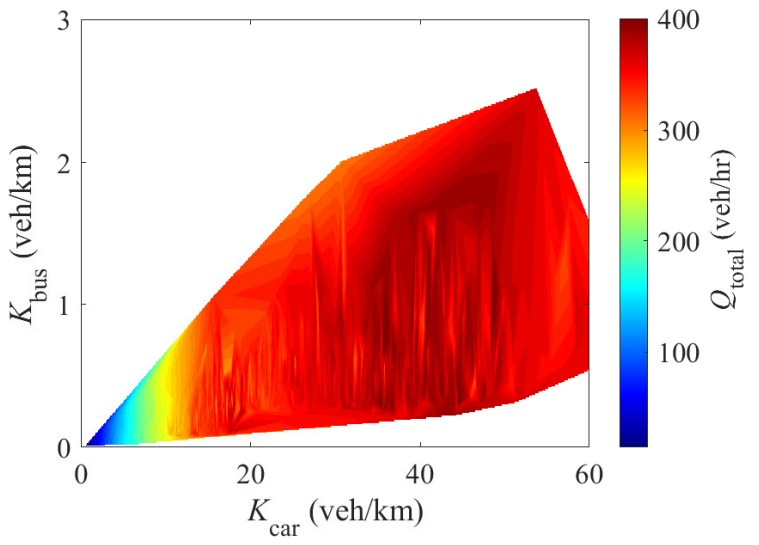

(b)

Fig. 6: (a) and (b) The 3D-NMFD of the Christchurch CBD and corresponding contour plot, respectively.

bus stop close to the trip generator center. Consequently, we may assume that a combination of the far-side and near-side bus stops (for bus stops that need to be located near to the intersections) leads to the optimal performance of the urban networks. Therefore, the current condition of the network is also compared with NSS and FSS.

Fig. 6. and Fig. 7. depict the 3D-NMFDs and corresponding contour plots for the current condition and NSS and FSS, respectively. In the free flow condition, all scenarios perform almost identical at car densities less than $17 \mathrm{veh} / \mathrm{km}$, but the FSS serves more buses in the higher densities. This scenario also results in a larger capacity range, in comparison with the NSS and current condition. This means that there is a bigger set of the car density $(33-53 \mathrm{veh} / \mathrm{km}$ ) at which the network with the FSS performs near to its capacity (deep red colour in the contour plot). However, the network under the current condition serves more buses in its capacity range. In addition, the network with the FSS does not experience the congested regime and remains in the capacity state. Instead, the congested regime appears in the current condition and the network with the NSS at the car density of $51 \mathrm{veh} / \mathrm{km}$. The results yield that, if the main concern is network performance, the far-side bus stops are superior to the nearside bus stops at the network level. This is in consistent with local level studies like $[6,8,11]$ in which authors presented that the far-side bus stops are generally preferable than the near-side bus stops. It is also a confirmation for the results presented by [34] which discussed the near-side and far-side bus stops at the network level using a toy grid network. Now, let us discuss the potential explanation of the presented outputs in our study. The reason behind this might be the fact that the near-side bus stops in particular cause more interaction between the car traffic and the public transport system. Specifically, in the peak periods when the queue of cars appears in the red phase of the traffic signal. We think that the interaction between the cars in the queue and the bus which needs to enter or exit the bus stop might negatively affect the car traffic delay and consequently the network performance. To examine the accuracy of this claim, the box plot of the average delay of car traffic in the entire network (for all 15 replications) is plotted for all scenarios see Fig. 8. In the box plot, the black bars show the minimum and maximum, bottom and top of the blue box depict the first and third quartile, respectively, and the red line shows the median. Also, the employed network average delay is the output of the Aimsun calculated as the difference between the expected travel time (in an ideal condition) and the travel time (with the unit of time per vehicle per kilometer). Then, the average of all vehicles' delays is introduced as the network average delay (where the unit is converted into time per kilometre). The car traffic experiences the lowest median in the current condition followed by the FSS while the NSS shows the highest median see Fig. 8a. This confirms what we discussed as the potential reason behind the better performance of the network with the FSS in comparison with NSS. Besides, in the case of the observed range (i.e. the distance between the max and min values in the box plot) in the average delay of car traffic, the scenarios almost perform identically. However, the max and min values are lower in the case of the current condition. The max value might be introduced as the worst case that the bus has to stop for the red phase in the case of FSS and has to wait in the car queue before entering the bus stop and stop for the red phase after exiting the bus stop in the case of NSS. This means that, in the worst case of the NSS, there is more interaction between the car traffic and public transport system compared to the worst case of the FSS. Therefore, it makes sense that FSS leads to a lower max value for car traffic compared to the NSS. In the case of min value, we may define it as the optimal cases that the buses arrive at the green phase for both FSS and NSS. The cars behind a bus have to decrease their speed when a bus wants to enter/exit the bus stop. This, in the case of the NSS, might result that some cars lose the green phase. Therefore, car traffic might experience a lower min value in the FSS scenario. The observed trend is expectable in the case of the NSS and FSS, but the better performance of the current condition compared to NSS and FSS confirms the assumption that we presented at the beginning of this subsection. Note 


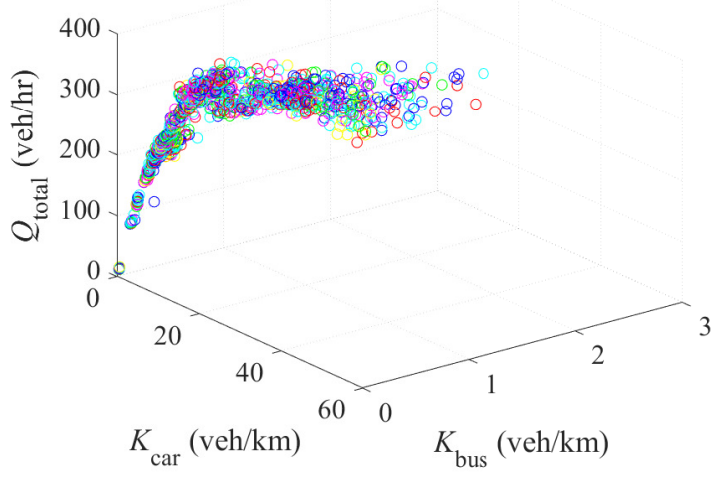

(a)

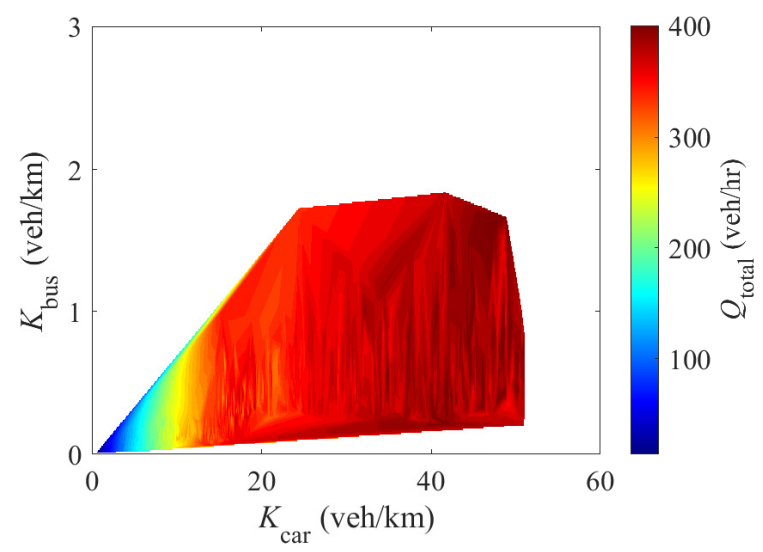

(b)

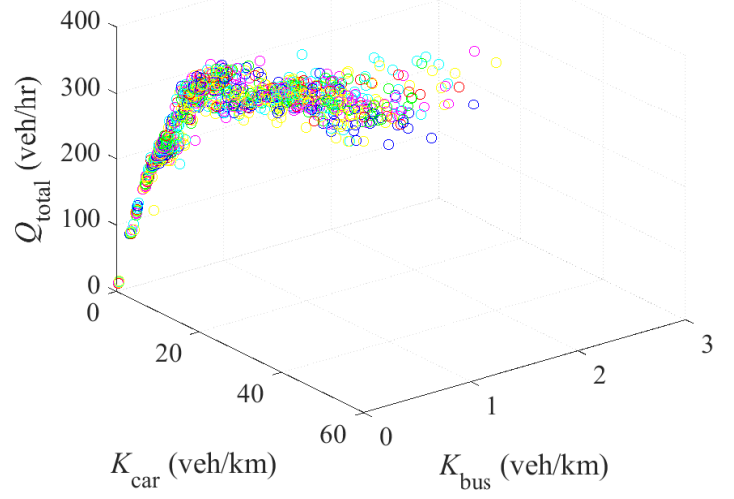

(c)

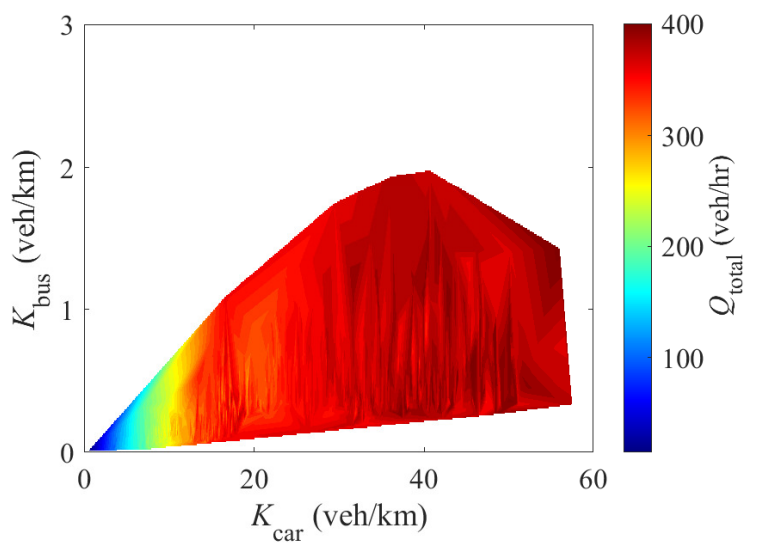

(d)

Fig. 7: (a) and (b) the 3D-NMFD and contour plot of the FSS, (c) and (d) the 3D-NMFD and contour plot of the NSS.

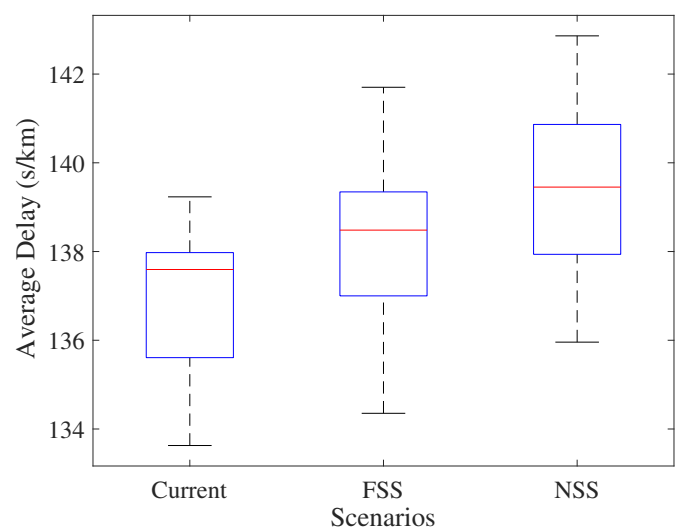

(a)

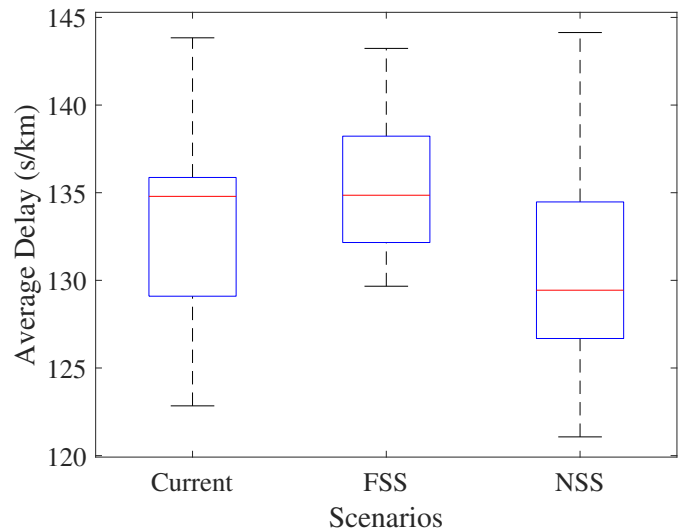

(b)

Fig. 8: (a) The network average delay of the car traffic, (b) The network average delay of the public transport system.

that, in addition to the network geometry limitation which resulted in having a few numbers of near-side bus stops in the FSS, the observed results show that, the considered criteria and the applied guideline in designing the bus stops location also affect the network performance and the average delay of the car traffic. Therefore, we can conclude that a combination of the near-side and far-side bus stops results in a better performance of the network and the network design properties affect this combination. Nevertheless, as we discussed in the background section, some local level studies like [25] presented that the near-side bus stop is superior if the main concern is the public transport system 
performance. This results in an interesting question at the network level that: what if the public transport performance is the main concern instead of the network performance? To answer it, the box plot of the network average delay of the public transport system (for all 15 replications) is plotted for all scenarios Fig. 8b. The NSS results in the lowest median for the public transport system. This is reasonable because near-side bus stops decrease the probability of double stops for buses. In terms of the observed range, it is clear that the FSS leads to the lowest observed range followed by the current condition. This happens because the far-side bus stops lead to a smoother interaction between the car traffic and public transport system and consequently a more reliable performance of the network. In the case of min values, the NSS scenario results in the lowest min value. This occurs since the possibility of arriving at the green phase for all the buses in the FSS scenario is almost impossible. It is worth mentioning that the average delay here is the network average delay, consequently, the NSS results in a lower min value. Therefore, reasonably, the min value increases as the number of far-side bus stops raise. Besides, the NSS scenario shows the highest max value followed by the current condition which is in consistent with the defined worst case. This means that, if the main concern is the public transport system performance, the near-side bus stops are superior at the network level which is in consistent with the local level studies like [25]. Besides, this comparison reveals that (i) the $\mathrm{CBD}$ of the city of Christchurch contains a well-designed public transport network which performs close to the FSS from the network performance point of view and even better than the FSS in case of the car traffic average delay, (ii) the public transport network design is almost in favor of the car traffic. Herein, the questions that raise and need to be answered are: what is the optimal combination of the bus stop location in a public transport network ? what criteria should be considered to achieve the optimal combination? How the network properties and the network stakeholders preferences affect this optimal combination? and finding the answer of this question has shaped a part of on-going research of the authors. Last but not least, this shows that, in addition to performance studies, the notion of 3D-NMFD can be also employed to improve the public transport network design works.

\section{Number of berth}

The comparison of the network performance under different berth number scenarios is provided in Fig. 9. The general observed trend yields that as the number of berth increases the capacity (range) increases which is in consistent with the local level studies like [28]. Also as we expect, the network capacity experiences a significant drop in the $B 1 S$. Indeed, the capacity decreases to $340 \mathrm{veh} / \mathrm{hr}$ with the car critical capacity range of (20-40 veh/ km) and the congested regime appears in the network. The reason behind this is the bottleneck which is activated by the bus that waits on the street to enter the bus stop when the bus stop is occupied with another bus. With increasing the berth number to two in the bus stops which serve two or more bus lines in the $B 2 S$, the network capacity increases to $400 \mathrm{veh} / \mathrm{km}$. The lower bound and upper bound of the critical density range of the car traffic also raise to 35 and $45 \mathrm{veh} / \mathrm{km}$, respectively. However, the network still experiences the congested regime. In the $B 3 S$ the network capacity remains about $400 \mathrm{veh} / \mathrm{km}$. But interestingly, the set of car density and bus density points at which the network performs close to its capacity (i.e. capacity range) dramatically increases in comparison to the $B 2 S$. The rise in the capacity range, which is the consequence of the raise in berth numbers in $B 3 S$, seems to match our expectations. However, the congested regime still appears in this scenario even more extreme in comparison to the $B 2 S$. Such an observation might have roots in the fact that some of the bus stops are located closer to the intersections in the B3S due to the applied increase in their lengths. This negatively affects the network performance and leads to more interactions between car traffic and the public transport system. Moreover, we expect that this affects the time delay of the car and bus in the network case of the $B 3 S$. It was also presented in the literature that the berth number affects the average delay of the public transport system [27]. Therefore, the box-plot of the average delay of the car traffic and public transport system in the entire network (for all 15 replications) are plotted for all scenarios see Fig. 10. The median experiences a reduction as berth number increases for both car and bus. In case of the car traffic, it is reasonable since with increasing the berth number the probability of bottleneck (i.e. the bottleneck created with the bus that has to wait outside of the bus stop due to the lac of berth number) activation significantly reduces. The results in the case of the public transport system are also in consistent with the local level literature [22]. Also, the observed range in the network average delay generally drops from $B 1 S$ to $B 3 S$ for both car traffic and public transport system due to the reduction in the bottleneck activation probability. Besides, if we define the min and max values as the optimal and worst cases when there are/are not activated bottlenecks, then we expect that both values decrease from $B 1 S$ to $B 3 S$. The expected trend almost occurs from B1S to B2S for both modes. However, from $B 2 S$ to $B 3 S$, the min values for both mode and max value of the public transport system increase and the max in the case of car traffic almost reminds the same. This is in consistent with the observed congestion in B3S that occurs due to the reduction in the bus stop distance from the intersection. The observed results in B3S can be highlighted as (i) the increase in the number of berths raises the network capacity ranges; (ii) the bus stops which are located closer to the intersections due to the applied increase in their lengths in B3S might result in the unexpected congestion or delay at the network level. While the first observation is expectable, the later means that the distance from intersection needs to be considered when designing the berth number in the bus stops which are located close to intersections. 


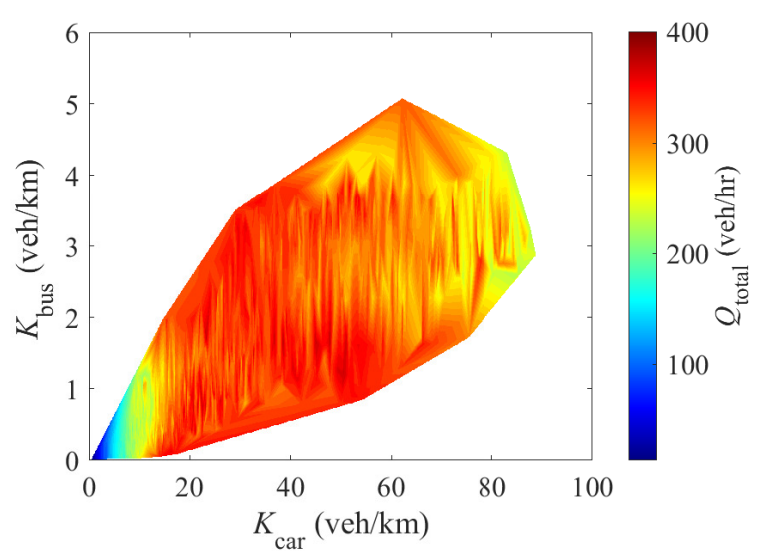

(a)

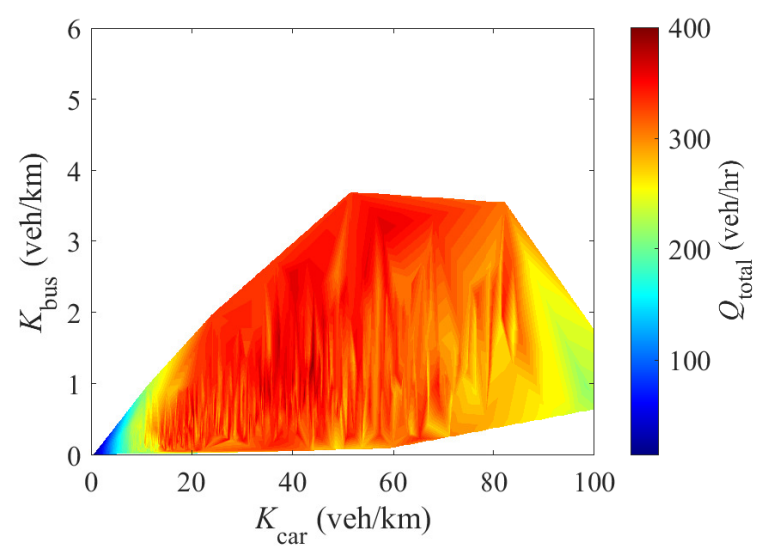

(c)

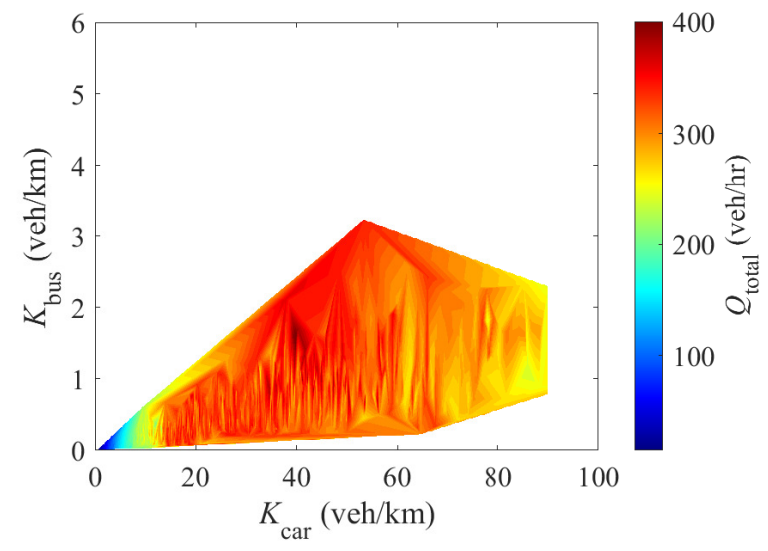

(b)

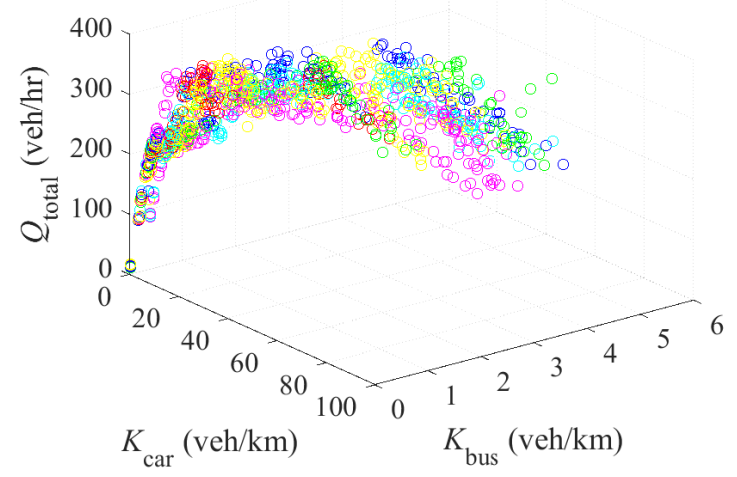

(d)

Fig. 9: (a), (b), and (c) depict the contour plot of the 3D-NMFDs of the $B 1 S, B 2 S$, and $B 3 S$, respectively, (d) the 3D-NMFD of the $B 1 S$ (worst case scenario). Note, the 3D-NMFD of the $B 2 S$, and $B 3 S$ are not entered due to efficient usage of the space.

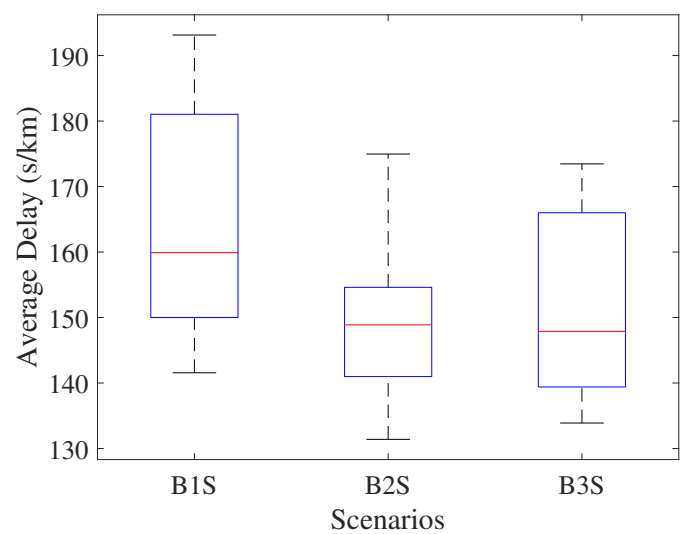

(a)

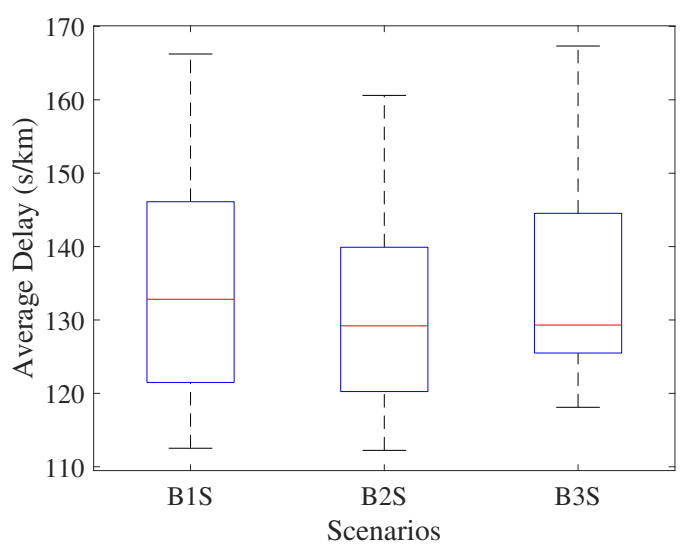

(b)

Fig. 10: (a) The network average delay of the car traffic, (b) The network average delay of the public transport system.

\section{CONCLUSION}

The impacts of operational characteristics of the public transport system on the network performance and the public transport system have been widely investigated in the lit- erature. However, the literature mainly includes local level studies. In addition, some local level studies with the same main concerns resulted in the opposite directions. For instance, [12] presented that the far-side bus stops are superior if the main concern is the delay of public transport system, 
while [15] recently proposed that the near-side bus stops are superior if the bus-carrying capacity is the major concern. Nevertheless, we think that the existence of the 3D-NMFD $[29,30]$ and its sensitivity to the operational characteristics of the public transport system provide a suitable platform to discuss the effects of these characteristics at the network level. Also, we think that the network level investigation of these impacts provides useful insight into studies that aim to design the public transport network.

In this paper, we discussed the effects of the bus stop location and berth number on the car traffic and public transport system at the network level. To this end, the notion of 3D-NMFD has been employed. We have shown through the simulation experiments that:

- The far-side bus stops (i.e. FSS) result in better network performance in terms of (i) the capacity range (i.e. the set of the car density and bus density at which network performs close to it capacity) and (ii) the critical density range at the network level.

- The near-side bus stops (i.e. NSS) lead to the lowest median in the box plot of the network average delay for the public transport system. However, the far-side bus stops (i.e. FSS) result in a lower median (compared to $N S S$ ) for the car traffic.

- The rise in the berth number increases the network capacity (range) and decreases the median in the network average delay box plot (see Fig.10) for both the car traffic and public transport system.

- The changes in the distance of the bus stop from intersection due to the increase in the berth number (the length of the bus stop) has to be considered to discuss the effects of the berth number on network performance.

- In real urban networks a combination of the near-side and far-side bus stops (i.e. bus stops that need to be located near to the intersections) might result in a better performance of the network.

The observed results are in consistent with the local level studies in both cases: the bus stop location $[6,8,11,15]$ and berth number $[17,18]$. The output about the effects of the far-side bus stops on the network performance confirms the presented results by [34] which was carried out for a toy grid network. Also, the proposed method and results can provide useful insights for practitioners and the organizations which aim to examine the performance of the public transport system and its effects on the network performance. The studies which aim to design the public transport system, specifically those that employ the notion of the NMFD like [5], also can take advantage of the provided results.

It is worth mentioning that, the provided investigation can also be carried out through a passenger-oriented perspective. For instance, the delay investigation can be carried out discussing delay per passenger instead of the vehicle delay. Also, the notion of the passenger 3D-NMFD can be employed instead of the vehicular 3D-NMFD which has been applied in our study. The passenger 3D-NMFD can be derived through Equation 4. Herein, assuming a constant car passenger occupancy 1.37 (per/veh) [43], the passenger 3D-NMFD of the Christchurch CBD is depicted in Fig.11. In the case of bus passenger occupancy, similar to the applied approach in [44], two different bus passenger occupancies are considered to mimic the effects of the passenger demand on the bus passenger occupancy ( $h_{b}$ is considered 10 (per/veh) in (7:00-8:00 AM) and 17 (per/veh) in (8:00-9:00 AM) [45]). As the first remark, the network experiences a capacity range of (550-600 per/hr) at the car density range of (35-50 $\mathrm{veh} / \mathrm{km})$ and bus density range of $(0.5-2 \mathrm{veh} / \mathrm{km})$. Besides, the general trend is similar to the observed vehicular 3DNMFD in Fig.6a. However, a more detailed investigation can be conducted via plotting the corresponding contour plots for different scenarios which is out of the scope of the present work.

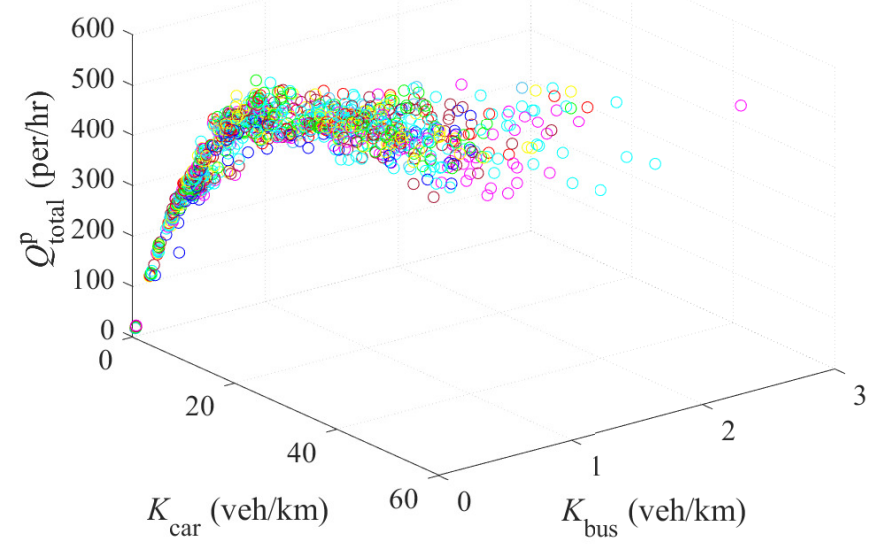

Fig. 11: Passenger 3D-NMFD of the Christchurch CBD.

A limitation of this study is that, in the case of the bus stop location, the considered scenarios are extreme cases. However, in a real urban network, it is not possible to have only far-side or near-side bus stops. We think that a combination of the near-side and far-side bus stops results in the best performance of the network. Initial insights of this assumption are provided in this study and it shapes the future research direction which is finding the optimal combination of the near-side and far-side bus stops in the network in order to optimize the network performance. In addition, it is worth noticing that the passenger activities and their interactions with the car traffic and public transport system are not simulated in this study. Another potential research direction can be the application of the notion of 3D-NMFD in public transport design studies. Also, the proposed method can be applied to investigate the effects of the other operational characteristics of the public transport 
system like the number of the bus line and bus stop type on network performance. Finally, more theoretical and empirical investigation is crucial to generalize the presented results even though the findings are in consistent with the local level studies and the current knowledge based on concepts in traffic flow theory.

\section{ACKNOWLEDGMENTS}

Special thanks go to Hee Seung Lee and Kyungrok Oh for their efforts to model the Christchurch CBD in the Aimsun environment at the Complex Transport System Laboratory (CTSLAB), University of Canterbury. The paper also benefited from the helpful and insightful comments of the three anonymous referees.

\section{REFERENCES}

[1] Daganzo, C.F.:'Structure of competitive transit networks', TRPart B: Methodological, 2010, 44, (4), pp 434-446

[2] Estrada, M., Roca-Riu, M., Badia, H., et al.:'Design and implementation of efficient transit networks: Procedure, case study and validity test', TRPart A: Policy and Practice, 2011, 9, (45), pp 935-950

[3] Badia, H., Estrada, M., Robuste, F.:'Competitive transit network design in cities with radial street patterns', TRPart B: Methodological, 2014, 59, pp 161-181

[4] Badia, H., Estrada, M., Robuste, F.:'Bus network structure and mobility pattern: A monocentric analytical approach on a grid street layout', TRPart B: Methodological, 2016, 93, pp 37-56

[5] Amirgholy, M., Shahabi, M., Gao, H.O.:'Optimal design of sustainable transit systems in congested urban networks: A macroscopic approach', TRPart E: Logistics and Transportation Review, 2017, 103, pp 261-285

[6] Fitzpatrick, K., Hall, K., Perdinson, D., et al. : 'Guidelines for the location and design of bus stops, Transit Cooperative Research Program Report 19' (TRB, 1996)

[7] Kittelson \& Associates and Transit Cooperative Research Program and Transit Development Corporation. : 'Transit capacity and quality of service manual' (TRB, 2003)

[8] Furth, P.G., SanClemente, J.L.:'Near side, far side, uphill, downhill: impact of bus stop location on bus delay', Transportation Research Record, 2006, 1971, 1, pp 66-73

[9] 'Manual, Highway Capacity' (TRB, 2000)

[10] Gu, W., Cassidy, M.j., Gayah, V.V., et al.:'Mitigating negative impacts of near-side bus stops on cars', TRPart B: Methodological, 2013, 47, pp 42-56

[11] Zhao, X., Gao, Z., Jia, B.:'The capacity drop caused by the combined effect of the intersection and the bus stop in a CA model', Physica A: Statistical Mechanics and its Applications, 2007, 385, 2, pp 645-658

[12] Gu, W., Gayah, V.V., Cassidy, M.J., et al.:'On the impacts of bus stops near signalized intersections: Models of car and bus delays', TRPart B: Methodological, 2014, 68, pp 123-140

[13] Gayah, V.V., Ilgin Guler, S., Gu, W.:'On the impact of obstructions on the capacity of nearby signalised intersections', Transportmetrica B: Transport Dynamics, 2016, 4, 1, pp 48-67

[14] Liu, Z., Jian, M.:'Traffic impacts analysis of bus stops near signalized intersections based on an optimal velocity model', Advances in Mechanical Engineering, 2019, 11, 5

[15] Shen, M., Gu, W., Hu, S., et al.:'Capacity approximations for near-and far-side bus stops in dedicated bus lanes', TRPart B: Methodological, 2019, 125, pp 94120

[16] Li, H., Mao, B., Bertini, R.L.:'Evaluating the impacts of bus facility design features on transit operations in Beijing, China: A simulation approach', 87th annual meeting of the transportation research board, Washington, D.C., USA, January 2008, pp 113-118

[17] Lu, L., Su, Y., Yao, D., et al.:'Optimal design of bus stops that are shared by multiple lines of buses', 13th International Conference on Intelligent Transportation Systems (IEEE-ITSC), Madeira Island, Portugal, September 2010, pp 125-130

[18] Yang, X., Si, B., Huan, M.:'Mixed traffic flow modeling near Chinese bus stops and its applications', Journal of Central South University, 2012, 19,9, pp 2697-2704

[19] Liu, L., Mao, B., Liang, X., et al.:'Study of the Effect of Queuing at Curbside Bus-Stop on Road Link Capacity', 15th COTA International Conference of Transportation Professionals, Beijing, China, July 2015, pp 1451-1459

[20] Godfrey, J.: 'The mechanism of a road network', Traffic Engineering \& Control, 1969, 8, (8)

[21] Herman, R., Prigogine, I.:'A two-fluid approach to town traffic', Science, 1979, 204, (4389), pp 148-151

[22] Mahmassani, H.S., Williams, J., Herman, R.:'Investigation of network-level traffic flow relationships: some simulation results', Transportation Research Record, 1984, 971, pp 121-130

[23] Geroliminis, N., Daganzo, C.F.:'Existence of urbanscale macroscopic fundamental diagrams: Some experimental findings', TRPart B: Methodological, 2008, 42, (9), pp 759-770

[24] Daganzo, C.F., Geroliminis, N.:'An analytical approximation for the macroscopic fundamental diagram of urban traffic', TRPart B: Methodological, 2008, 42, (9), pp 771-781

[25] Lu, S., Knoop, V.L., Keyvan-Ekbatani, M.:'Using taxi GPS data for macroscopic traffic monitoring in large scale urban networks: calibration and MFD derivation', Transportation research procedia, 2018, 34, pp 243-250

[26] Corman, F., Henken, J., Keyvan-Ekbatani, M.:'Macroscopic fundamental diagrams for train operations-are we there yet?', 6th International Conference on Models and Technologies for Intelligent 
Transportation Systems (MT-ITS), Krakow, Poland, June 2019, pp 1-8

[27] Keyvan-Ekbatani, M., Gao, X., Gayah, V., et al.:'Traffic-responsive signals combined with perimeter control: investigating the benefits', Transportmetrica B: Transport Dynamics, 2019, 7, (1), pp 1402-1425

[28] Boyac, B., Geroliminis, N.:'Estimation of the network capacity for multimodal urban systems', ProcediaSocial and Behavioral Sciences, 2011, 16, pp 803-813

[29] Geroliminis, N., Zheng, N., Ampountolas, K.:'A threedimensional macroscopic fundamental diagram for mixed bi-modal urban networks', TRPart C: Emerging Technologies, 2014, 42, pp 168-181

[30] Loder, A., Ambühl, L., Menendez, M., et al.:'Empirics of multi-modal traffic networks-Using the 3D macroscopic fundamental diagram', TRPart C: Emerging Technologies, 2017, 82, pp 88-101

[31] Loder, A., Dakic, I., Bressan, L., et al.:'Capturing network properties with a functional form for the multimodal macroscopic fundamental diagram', TRPart B: Methodological, 2019, 129, pp 1-19

[32] Ortigosa, J., Zheng, N., Menendez, M., et al.:'Analysis of the 3D-vMFDs of the urban networks of Zurich and San Francisco', 18th International Conference on Intelligent Transportation Systems (IEEE-ITSC), Canary Islands, Spain, September 2015, pp 113-118

[33] Castrillon, F., Laval, J.:'Impact of buses on the macroscopic fundamental diagram of homogeneous arterial corridors', Transportmetrica B: Transport Dynamics, 2018, 6, 4, pp 286-301

[34] Johari, M., Keyvan-Ekbatani, M., Ngoduy, D., et al.:'Effects of Near-Side and Far-Side Bus Stops on NMFD of Bi-Modal Urban Network', 2019 IEEE Intelligent Transportation Systems Conference (ITSC), Auckland, New Zealand, October 2019, pp 746-751

[35] Keyvan-Ekbatani, M., and Kouvelas, A., Papamichail, I., et al.:'Exploiting the fundamental diagram of urban networks for feedback-based gating', TRPart B: Methodological, 2012, 46,10, pp 1393-1403

[36] Saberi, M., Mahmassani, H.S., Hou, T., et al.:'Estimating network fundamental diagram using three-dimensional vehicle trajectories: Extending edie's definitions of traffic flow variables to networks', Transportation Research Record, 2014, 2422,1, pp $12-20$

[37] 'TSS transport simulation systems, aimsun users manual version 8 , barcelona, spain' (2014)

[38] 'CAST v18a, Model Update Summary Report' (May, 2019)

[39] 'SATURN: Version 11 Manual' (2015)

[40] 'Metro Information', http://m.metroinfo.co.nz/, accessed 8 November 2019

[41] Keyvan-Ekbatani, M., and Papageorgiou, M., Knoop, V.L. :'Controller design for gating traffic control in presence of time-delay in urban road networks', TRPart C: Emerging Technologies, 2015, 59, pp 308-322

[42] Fernández, R.:'Modelling public transport stops by microscopic simulation', TRPart C: Emerging Technologies, 2010, 18,6, pp 856-868

[43] Sullivan, Ch., Carolyn, O.:'Vehicle occupancy in New Zealand's three largest urban areas', Leading Transport Research in the 21st Century Conference, Wellington, New Zealand, 2003,

[44] Haitao, H., Yang, K., Liang, H., et al.:'Providing public transport priority in the perimeter of urban networks: A bimodal strategy', TRPart C: Emerging Technologies, 2019, 107, pp 171-192

[45] 'Central Area Bus Operational Review, Final Report' (November, 2009) 